\title{
Comment on "Resistance training alone reduces systolic and diastolic blood pressure in prehypertensive and hypertensive individuals: a meta-analysis"
}

\author{
Odilon Abrahin ${ }^{1,2}$ - Evitom Corrêa de Sousa ${ }^{1,3,4,5} \cdot$ Ana Lorena Lima Ferreira ${ }^{1} \cdot$ Rejane Pequeno Rodrigues ${ }^{1,2}$. \\ Erik Artur Cortinhas Alves ${ }^{1} \cdot$ Rodolfo Paula Vieira ${ }^{3,4,5}$
}

Received: 20 July 2018 / Revised: 23 July 2018 / Accepted: 23 July 2018 / Published online: 5 November 2018

(c) The Japanese Society of Hypertension 2018

\section{In Response:}

We thank Drs. Khazaei, Karami, and Veisani for their interest in our research. We will address their questions and comments in the order presented in the letter to the editor.

Our study included five electronic databases: PubMed, Cochrane Library, LILACS, BIREME, and SciELO. Unfortunately, we cannot include all electronic databases, but we appreciate the suggestion and will use Web of Science in future studies. If Drs. Khazaei, Karami, and Veisani have found any additional studies that may be included in our meta-analysis (according to our inclusion criteria), please inform us. We intend to increase the number of studies and the analytical power in our next meta-analysis.

In our meta-analysis, we included only randomized controlled trials (RCT), prehypertensive, and hypertensive subjects, and the practice of isotonic resistance training alone. These inclusion criteria considerably increase the quality of the articles but decrease the number of included

Odilon Abrahin

odilonsalim@hotmail.com

1 Laboratory of Resistance Exercise and Health, Campus III, Department of Physical Education, Pará State University, Belém, Brazil

2 Faculdade Metropolitana da Amazônia-FAMAZ, Belém, Brazil

3 University Brazil, Post-graduation Program in Bioengineering, São Paulo, SP, Brazil

4 Brazilian Institute of Teaching and Research in Pulmonary and Exercise Immunology (IBEPIPE), São José dos Campos, SP, Brazil

5 Federal University of São Paulo (UNIFESP), Post-graduation Program in Sciences of Human Movement and Rehabilitation, Santos, SP, Brazil studies compared with other meta-analyses $[1,2]$ on this topic.

Finally, our meta-analysis was reported in accordance with the recommendations and criteria outlined in the preferred reporting items for systematic reviews and metaanalysis (PRISMA) statement. The two reviewers independently evaluated the full-text articles and performed data extraction. In addition, the heterogeneity was adequate, with $I^{2}: 22.5 \%$ and a $P$-value for heterogeneity $=$ 0.271 for systolic blood pressure, and $I^{2}: 46.5 \%$ and a $P$-value for heterogeneity $=0.113$ for diastolic blood pressure.

We thank Drs. Khazaei, Karami, and Veisani for their interest in our study and hope we have addressed their concerns. As before, we propose to update our metaanalysis by adding new studies.

\section{Compliance with ethical standards}

Conflict of interest The authors declare that they have no conflict of interest.

\section{References}

1. MacDonald HV, Johnson BT, Huedo-Medina TB, Livingston J, Forsyth KC, Kraemer WJ, et al. Dynamic resistance training as stand-alone antihypertensive lifestyle therapy: A meta-analysis. J Am Heart Assoc. 2016;28:e03231.

2. Lemes ÍR, Ferreira PH, Linares SN, Machado AF, Pastre CM, Netto J. Jr. Resistance training reduces systolic blood pressure in metabolic syndrome: a systematic review and meta-analysis of randomised controlled trials. Br J Sports Med. 2016;50: $1438-42$. 